\title{
Alterations in Rat Renal Glucose Transport following in Vivo use of Freund's Adjuvant
}

\author{
AARON L. FRIEDMAN, SUSAN LANGAN, TERRY OBERLEY, AND MARK THORNQUIST \\ Departments of Pediatrics, Pathology and Statistics, University of Wisconsin, Madison, Wisconsin 53792
}

\begin{abstract}
Alterations in rat renal glucose transport following in vivo use of Freund's adjuvant were examined. Lewis-Brown Norway rats were placed in four separate injection groups: 1) tubular basement membrane plus adjuvant [complete Freund's adjuvant (CFA) plus pertussis]; 2) adjuvant (CFA plus pertussis); 3) CFA only; or 4) pertussis only. Renal handling of glucose was assessed 14 days after a single injection. No in vivo changes were noted. No histologic differences among groups were noted. However, brush border membrane vesicles prepared from animals in groups 1, 2, and 3 showed a marked decrease in glucose uptake. Further, Michaelis-Menten kinetics demonstrated a decrease in apparent $\mathrm{Km}$ and $\mathrm{Vmax}$ for glucose in groups 1, 2, and 3. CFA alone can cause a change in brush border membrane vesicle uptake of glucose. The pathogenic mechanism behind CFA-induced transport changes remains unclear. However, studies employing CFA cannot dismiss Freund's adjuvant as "inert" and must take into account functional changes created by CFA alone. (Pediatr Res 20: 443-446, 1986)
\end{abstract}

\section{Abbreviations}

CFA, complete Freund's adjuvant

L-BN, Lewis-Brown Norway

BBMV, brush border membrane vesicles

TBM, tubular basement membrane

THM, tris-hepes-mannitol

$\mathrm{S}$, supernate

$P$, pellet

Freund's adjuvant has been used extensively in immunologic study. First described in the 1940 s as a method of enhancing delayed hypersensitivity (1), Freund's adjuvant has become a standard experimental tool to enhance the immunologic response. CFA, which is composed of paraffin oil and killed mycobacterium, has been employed as part of the induction technique in experimental glomerulonephritis (2-5).

Recently, Miettinen (6) has shown that rabbits injected only with Freund's adjuvant produce antirenal brush border antibodies. In this report, we provide evidence for functional derangement in proximal tubule transport as a result of the in vivo administration of adjuvant in the rat.

\section{METHODS}

L-BN rats (Microbiological Associates, Walkersville, MD) weighing 200-250 g were injected subcutaneously with one of five solutions: 1) TBM plus adjuvant-approximately $0.5-1 \mathrm{mg}$

Received July 1, 1985; accepted January 7, 1986.

Correspondence Aaron L. Friedman, M.D., Department of Pediatrics, University of Wisconsin Hospitals, 600 Highland Avenue, Madison, WI 53792.

Supported by a grant from the Graduate School Research Committee of the University of Wisconsin. of TBM and $0.2 \mathrm{ml}$ of adjuvant; the adjuvant was composed of CFA (Sigma Pharmaceuticals, St. Louis, MO) plus pertussis antigen (Wyeth Laboratories, Philadelphia, PA); 2) adjuvant only- $0.2 \mathrm{ml}$ of CFA plus pertussis; 3) CFA only, $0.2 \mathrm{ml}$; 4) pertussis only, $0.2 \mathrm{ml}$; or 5) control- $0.2 \mathrm{ml}$ of $0.9 \% \mathrm{NaCl}$.

$T B M$ production. Tubular membrane material was prepared by separating cortex from medulla and then homogenizing the cortex in a Teflon-glass homogenizer with approximately $60-80$ $\mathrm{ml}$ of saline. It was centrifuged at $600 \mathrm{rpm}$ for $4 \mathrm{~min}$, and the supernatant was discarded. The pellet was washed again with 80 $\mathrm{ml}$ of saline and passed through an 80 -mesh screen and then 230 -mesh screen using approximately $200 \mathrm{ml}$ of saline to wash the material. The filtrate was centrifuged at $1500 \mathrm{rpm}$ for $5 \mathrm{~min}$. The supernatant was discarded, and the pellet was resuspended in $20 \mathrm{ml}$ of saline and sonicated with a Bronson sonicator (setting 3 ) for $45 \mathrm{~s}$. The sonicate was spun at $3000 \mathrm{rpm}$ for $10 \mathrm{~min}$, and the pellet was saved for use as tubule membrane antigen. The purpose of this treatment was to prepare immunizing antigen composed primarily of tubular membrane, with minimal glomerular contamination.

Twelve to 14 days following injection, animals were placed in metabolic cages. Twenty-four-h urine collections were made for creatinine clearance and glucose excretion. After blood was obtained for creatinine and glucose, the animals were sacrificed for in vitro studies.

Preparation of $B B M V$. BBMV were prepared by a modification of the method of Booth and Kenny (7). Kidney cortex was weighed and minced and then homogenized in THM buffer $(20: 1, \mathrm{v} / \mathrm{w})$ on ice using an Elvejhem/Potter homogenizer (Teflon on glass) inserted into a Wheaton overhead stirrer. The tissue was homogenized until no large pieces of tissue were observable (approximately 10 strokes at setting 3 ). The homogenate was next homogenized in a glass/glass homogenizer for about five strokes. Dry $\mathrm{CaCl}_{2}$ was added to the homogenate to give a final concentration of $10 \mathrm{mM}$. The mixture was stirred for $15 \mathrm{~min}$ on ice. An equal volume of THM- $10 \mathrm{mM} \mathrm{CaCl}$ was added to the mixture which was then centrifuged at $500 \times g$ for $12 \mathrm{~min}$.

The $S_{1}$ was saved, and the pellets were resuspended in $40 \mathrm{ml}$ of THM- $10 \mathrm{mM} \mathrm{CaCl}$ and respun at $500 \times g$ for $12 \mathrm{~min}$. The supernate was combined with $S_{1}$ and centrifuged at $15,000 \times g$ for $12 \mathrm{~min}$. The $\mathrm{P}_{2}$ was resuspended by gently aspirating through an 18-gauge needle in about $3 \mathrm{ml}$. It was then made up to the original 20:1 volume of THM. Dry $\mathrm{CaCl}_{2}$ to a concentration of $10 \mathrm{mM}$ was added, and the suspension was stirred on ice for 15 min. The $\mathrm{S}_{2}$ was spun once again at $15,000 \times g$, and the resulting pellet was suspended in a small volume (1-2 ml) of THM and $10 \mathrm{mM} \mathrm{CaCl}_{2}$ and added to the mixture on ice. After centrifuging at $750 \times g$ for $12 \mathrm{~min}$, the resulting $\mathrm{S}_{3}$ was centrifuged at 30,000 $\times g$ for 12 min. $\mathbf{P}_{4}$ contained the brush border vesicles and was resuspended in the original 20:1 volume of THM-300 (TH buffer with $300 \mathrm{mM}$ mannitol) to wash out the $\mathrm{CaCl}_{2}$. The resuspended $P_{5}$ was spun at $48,000 \times g$ for $20 \mathrm{~min}$, and the resulting $\mathbf{P}_{6}$ was resuspended in about $20 \%$ of original $20: 1$ volume in THM-300. The suspension was centrifuged at $2000 \times g$ for $5 \mathrm{~min}$, and the supernate was then spun at $48,000 \times g$ for $20 \mathrm{~min}$. 
$\mathbf{P}_{7}$ contained the purified BBMV. One gram of kidney cortex yielded approximately $7 \mathrm{mg}$ of protein in the final product. The membranes were resuspended in THM-300 to give a final protein concentration of about $3.5 \mathrm{mg} / \mathrm{ml}$.

Uptake studies with brush border membranes. Brush border uptake studies were carried out, as per previous studies (8), by incubating the membrane vesicles at room temperature in small test tubes with varying concentrations of "cold" glucose and ${ }^{14} \mathrm{C}$ glucose. Two types of experiments were done: 1) Time course: membrane vesicles were incubated in buffer with $60 \mu \mathrm{M}$ glucose, and samples were taken over a 45 -min period to measure uptake as a function of time. 2) Concentration-dependent uptake: This was used to determine initial rate kinetics (apparent $\mathrm{Km}$ and $V \max$ ). The ability of brush border to transport glucose was measured from $15 \mu \mathrm{M}$ to $1.5 \mathrm{mM}$ with a 15 -s incubation period. These two experiments measured the Na-dependent uptake, since the membranes were prepared in the absence of sodium, but the incubations were carried out in the presence of $100 \mathrm{mM}$ sodium. Uptake studies were also done in the absence of sodium to measure Na-independent uptake. Na-independent values were subtracted from Na-dependent values. Uptake was measured by applying the sample to a $0.22 \mu$ pore size filter and washing three times with $2 \mathrm{ml}$ of cold buffer. Suction was applied with a vacuum pump. All uptake studies were performed in triplicate. The filters were placed in scintillation vials and allowed to dry overnight in a $75^{\circ}$ oven. Scintillation fluid ( $4 \mathrm{~g} /$ liter PPO and 50 $\mathrm{mg}$ /liter POPOP in scintillation-grade toluene) was then added, and the samples were counted in a Beckman LS 7000 counter. Protein was determined by a modified method of Lowry et al. (9), and the DPM/mg protein was thus estimated.

Biochemical measurements. Plasma and urine creatinine were measured as per Sigma Technical Bulletin no. 555. Urines were first spun at $2000 \mathrm{rpm}$ for $10 \mathrm{~min}$ to remove particulate matter before any assays were done. Plasma and urine glucose were analyzed using the Beckman Glucose Analyzer 2. Brush border membrane purity and yield were checked by measuring: 1) protein, via modification of the Lowry procedure by Hartree $(10)$; 2) sodium-potassium ATPase, both total and ouabainsensitive (11); and 3) $\gamma$-glutamyl transpeptidase, as per Sigma Technical Bulletin no. 415. These enzyme assays represent a standard technique for verifying vesicle purity (12). $\gamma$-Glutamyl transpeptidase is a brush border marker, while sodium-potassium ATPase is a basolateral membrane marker.

Materials. ${ }^{14} \mathrm{C}$-glucose (specific activity $329 \mathrm{Ci} / \mathrm{mol}$ ) was purchased from New England Nuclear (Boston, MA). All solutions were prepared from reagent-grade chemicals. Milipore filters were purchased from Gilman Sciences (Ann Arbor, MI), and antirat IgG was obtained from Sigma Pharmaceuticals.

\section{HISTOLOGY}

Following removal of kidneys for in vitro study, a section of cortex was removed for histologic study. Standard hematoxylin and eosin sections were prepared for light microscopic evaluation. Fluorescent microscopic determination of $\mathrm{IgG}$ deposition was also performed using standard techniques. A small sample of kidney cortex was placed in glutaraldehyde and prepared by standard technique for electron microscopic evaluation. Slides were read in a "blind" fashion by a renal pathologist (T.O.). Small samples of BBMV from control and experimental groups were analyzed using standard electron microscopy techniques.

\section{ANALYSIS OF DATA}

Endogenous creatinine clearance was determined by standard clearance methods. Glucose excretion was expressed as $\mathrm{mg} / \mathrm{mg}$ creatinine. Time course of uptake assessment of Na-dependent uptake and determination of approximate point of equilibrium (net influx $=$ net efflux). $\mathrm{Km}$ was measured in $\mu \mathrm{mol} /$ liter and velocity in $\mu \mathrm{mol} / \mathrm{mg}$ protein/unit of time. $\mathrm{Km}$ and Vmax are frequently determined using the least-squares method. In order to draw valid inferences from least-squares regression, it is essential that the error variance at each value of the substrate concentration be the same. These data did not give uniform error variance at each substrate concentration. Thus, the more general technique of nonlinear least-squares regression was performed, with each data point given a weight based on its error variance. The regressions were performed using computer programs and the minitab statistical packages (13). Statistical significance was determined by Student's $t$ test and analysis of variance.

\section{RESULTS}

Review of the data revealed no statistical difference in in vivo results between any of the groups. Analysis of the in vitro data revealed no difference between group 4 (pertussis only) and group 5. These two groups are termed "control." Groups I (TBM plus adjuvant), 2 (adjuvant), and 3 (CFA only) were not statistically significantly different from one another. Taken separately each group was statistically different from groups 4 and 5 and these groups are termed "CFA-treated" animals. Groups 1, 2, and 3 taken separately were statistically different from groups 4 and 5 . Accordingly, CFA-treated animals (groups 1, 2, and 3) were grouped and termed CFA treated.

Histologic evaluation revealed no significant change in renal architecture between control and CFA-treated groups. Further, no significant cellular infiltrates were noted. Electron microscopy revealed no defects in renal tubular morphology and, in particular, no changes were noted at the brush border membrane. Evaluation of brush border vesicles by electron microscopy revealed no difference among any of the groups. Brush border vesicles appeared similar to those previously seen in our laboratory (8). Immunofluorescent study of tissue revealed linear deposition of IgG along tubular basement membrane in animals injected with TBM plus adjuvant (group 1), but control groups and animals injected with adjuvant, CFA, or pertussis only (groups 2, 3, and 4) showed no IgG deposition.

Endogenous creatinine clearances performed on animals during the 24-h period before sacrifice revealed no difference between control and CFA-treated animals (Table 1). Further, 24-h urinary excretion of glucose was determined, and no difference was seen in the urinary excretion or plasma levels of glucose between control and CFA-treated groups. Enzyme measurements for membrane purity are shown in Table 2. BBMV showed marked enhancement of luminal membrane fractions with no enrichment of basolateral membrane markers. No difference in enzyme purity was seen between control and CFA-treated animals. Table 2 shows no significant difference among any of the groups. When grouped into control and CFA-treated groups no difference could be determined. Control $\gamma$-glutamyl transpeptidase fold purification was $11.2 \pm 2.92$ while CFA-treated fold purification was $10.17 \pm 2.4$. No step up in Na-K-ATPase activity between homogenate and brush border membrane vesicles was seen in any of the five groups.

Uptake of glucose by BBMV demonstrated significant differences between control and CFA treated animals. Figure 1 shows the uptake of $60 \mu \mathrm{M}$ glucose into BBMV measured from $15 \mathrm{~s}$ to $8 \mathrm{~min}$. The graph depicts relative uptake compared to equilib-

Table 1. Creatinine clearance and glucose excretion (mean \pm $S E)$

\begin{tabular}{lcc}
\hline & Control* $^{*}$ & CFA treated $\dagger$ \\
\hline Endogenous creatinine & $1.46 \pm 0.28$ & $1.42 \pm 0.21$ \\
clearance $(\mathrm{ml} / \mathrm{min})$ & $(n=11)$ & $(n=18)$ \\
Plasma glucose $(\mathrm{mM})$ & $5.74 \pm 0.7$ & $5.49 \pm 0.78$ \\
& $(n=11)$ & $(n=18)$ \\
Urine & None detected & None detected \\
\hline
\end{tabular}

* Groups 4 and 5 .

$\dagger$ Groups 1, 2, and 3. 
Table 2. Brush border enzyme purity (mean \pm SE for specific activity)*

\begin{tabular}{lcl}
\hline & $\begin{array}{c}\gamma \text {-Glutamyl } \\
\text { transpeptidase }\end{array}$ & Na-K-ATPase \\
\hline Group 1 $(n=6)$ & & \\
H & $0.78 \pm 0.12$ & $0.139 \pm 0.03$ \\
B & $7.77 \pm 0.51$ & $0.147 \pm 0.03$ \\
Fold enrichment & $9.31 \pm 1.8$ & 1.2 \\
Group 2 $(n=6)$ & & \\
H & $0.93 \pm 0.11$ & $0.125 \pm 0.02$ \\
BB & $1.11 \pm 0.62$ & $0.125 \pm 0.03$ \\
Fold enrichment & $11.8 \pm 2$ & 1.1 \\
Group 3 $(n=6)$ & & \\
H & $1.11 \pm 0.21$ & $0.089 \pm 0.01$ \\
BB & $10.48 \pm 1.1$ & $0.092 \pm 0.01$ \\
Fold enrichment & $9.42 \pm 2.1$ & 0.95 \\
Group 4 $(n=5)$ & & \\
H & & \\
BB & $0.92 \pm 0.81$ & $0.096 \pm 0.009$ \\
Fold enrichment & $10.14 \pm 1.8$ & $0.096 \pm 0.008$ \\
Group 5 $(n=6)$ & $11.05 \pm 2.2$ & 1.0 \\
H & & \\
BB & $0.95 \pm 0.57$ & $0.143 \pm 0.016$ \\
Fold enrichment & $10.72 \pm 1.4$ & $0.136 \pm 0.011$ \\
\hline
\end{tabular}

* $\mathrm{H}$, homogenate; $\mathrm{BB}$, brush border vesicles; fold enrichment $(\mathrm{BB} / \mathrm{H})$; Control, groups 4 and 5; CFA-treated $=$ groups 1, 2, and 3 .

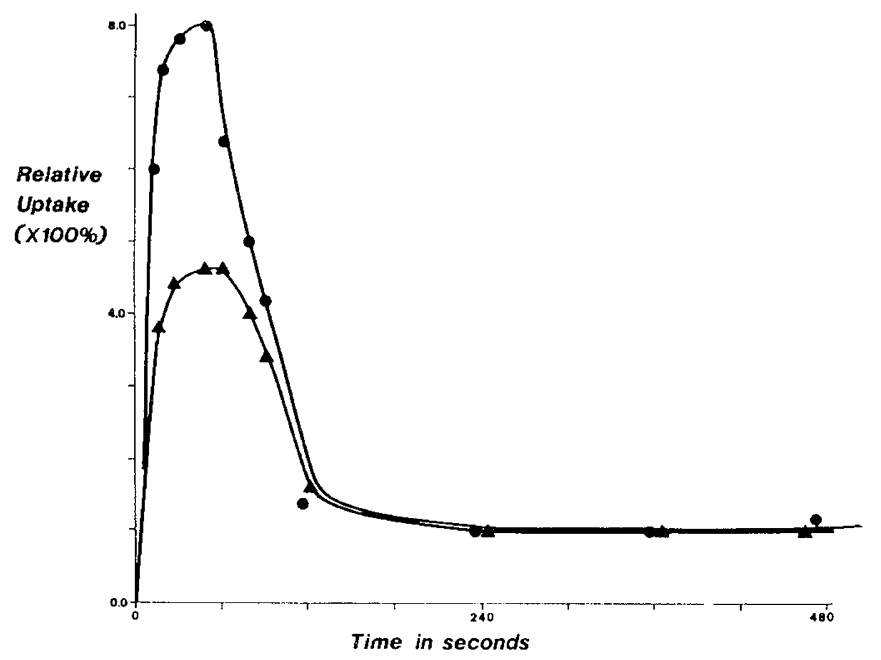

Fig. 1. Time course of uptake of glucose $(60 \mu \mathrm{M})$ into BBMV. Uptake is relative to equilibrium values. $(\mathrm{O}$, control groups 4 and $5 ; \triangle$, CFAtreated groups 1,2 and $3 ; \mathrm{SE}= \pm 9 \%$ )

rium. An 8-fold "overshoot" is noted in control animals with a blunted response seen in CFA-treated rats (4.5-fold overshoot). The difference in uptake was significant to $p<0.05$ from 15 to $90 \mathrm{~s}$. After $90 \mathrm{~s}$ no significant difference was noted. Table 3 demonstrates the actual uptake in pmoles glucose $/ \mathrm{mg}$ protein for the five groups. No statistical difference can be shown among groups 1, 2, and 3. Further groups 4 and 5 are not statistically different from one another. However, CFA treated (groups 1, 2, 3 ) are statistically different from control (groups 4 and 5) as shown in Table 3 .

Concentration-dependent uptake studies were performed over a glucose concentration range of $15 \mu \mathrm{M}$ to $1.5 \mathrm{mM}$. All uptake studies were done at $15 \mathrm{~s}$ of incubation. Figure 2 shows the velocity versus substrate concentration curve for control animals.
Table 3. Uptake of glucose by BBMV (pmol glucose/mg protein

\begin{tabular}{cccc}
\multicolumn{4}{c}{ $\pm S D)^{*}$} \\
\hline Time $(s)$ & \multicolumn{1}{c}{30} & \multicolumn{1}{c}{60} & \multicolumn{1}{c}{120} \\
\hline Group 1 $(n=6)$ & $523 \pm 94$ & $600 \pm 110$ & $180 \pm 30$ \\
Group 2 $(n=6)$ & $601 \pm 123$ & $584 \pm 91$ & $206 \pm 40$ \\
Group 3 $(n=6)$ & $574 \pm 100$ & $623 \pm 52$ & $160 \pm 47$ \\
Group 4 $(n=5)$ & $911 \pm 140$ & $950 \pm 141$ & $178 \pm 53$ \\
Group 5 $(n=6)$ & $956 \pm 157$ & $899 \pm 110$ & $194 \pm 68$ \\
\hline
\end{tabular}

* At 30 and $60 \mathrm{~s}$ groups 1,2 , and 3 different from groups 4 and $5 ; p$ $<0.05$.

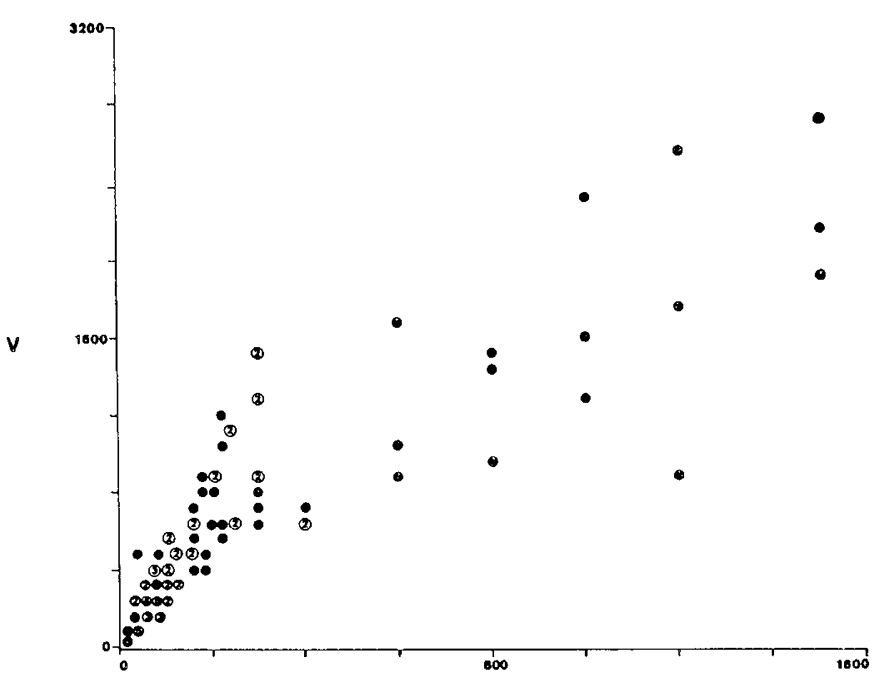

[s]

Fig. 2. Control, concentration-dependent uptake (V/S plot). $S$, glucose concentration varying from $15 \mu \mathrm{M}$ to $1.5 \mathrm{mM} ; V, \mathrm{pmol} / \mathrm{mg}$ protein/ $15 \mathrm{~s}$. Minimum of three experiments; all points are plotted.

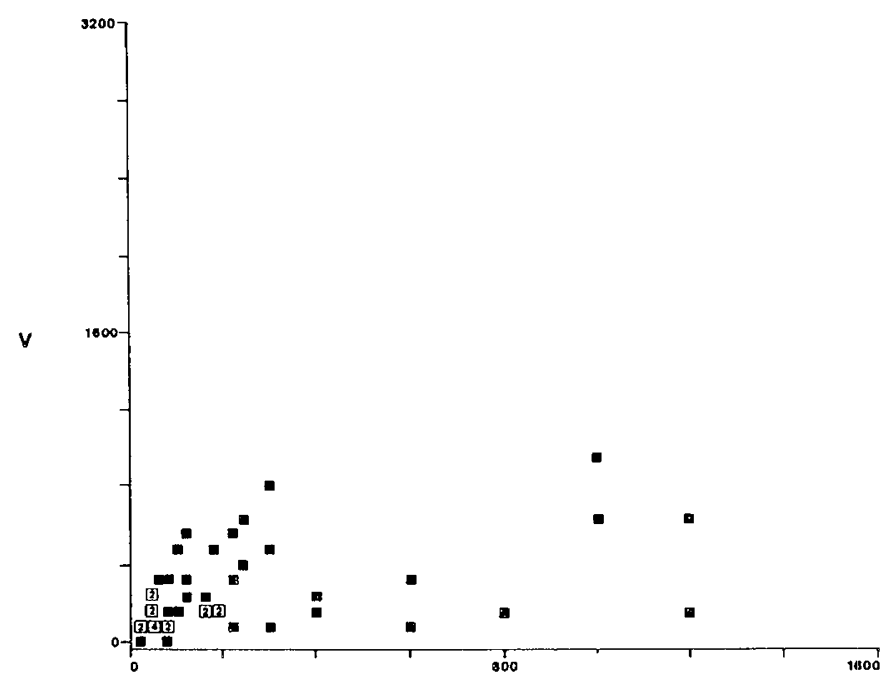

[s]

Fig. 3. CFA treated, concentration-dependent uptake (V/S plot). $S$, glucose concentration varying from $15 \mu \mathrm{M}$ to $1.5 \mathrm{mM} ; V, \mathrm{pmol} / \mathrm{mg}$ protein $/ 15 \mathrm{~s}$. Minimum of three experiments; all points are plotted.

Figure 3 depicts the same for CFA-treated animals. The CFAtreated animals show a "blunted" response at nearly all substrate concentrations. Apparent $\mathrm{Km}$ and Vmax were determined from the concentration-dependent uptake studies. $\mathrm{Km}$ for control versus CFA treated was $90 \pm 21$ (SD) versus $9 \pm 4 \mathrm{mmol} /$ liter glucose (control versus CFA treated $p<0.01$ ). Vmax for control 
versus CFA treated was $3225 \pm 621$ (SD) versus $392 \pm 79 \mathrm{pmol}$ glucose/mg protein $/ 15 \mathrm{~s}$ (control versus CFA treated $p<0.01$ ).

\section{DISCUSSION}

CFA has been used extensively in experimental studies of glomerulonephritis. In many experiments employing active immunization techniques, CFA is used. Steblay (4) produced antiglomerular basement membrane disease with heterologous or autologous glomerular basement membrane plus CFA. Heymann nephritis is induced with renal tubular antigen plus CFA (15). Further, the studies of anti-TBM antibody using active immunization also employed CFA $(15,16)$. The above and subsequent studies concentrated on the immunologic and histologic aspects of the experimental glomerulonephritis. Little information is available on the functional significance of many forms of immune-mediated renal disease and, in particular, little is known about the renal dysfunction caused by immune-mediated tubular disease.

Alteration in proximal tubule function with immune-mediated disease has been reported by Park et al. $(17,18)$. In these studies, changes in PAH transport into renal cortex slices following Heymann nephritis were documented. Freund's adjuvant was used as part of the immunization process. However, these studies did not discuss the effect of Freund's adjuvant alone on PAH transport.

To examine the functional significance of immune-mediated kidney disease using the active immunization model, an implicit assumption is that CFA does not in itself cause disease. However, Miettinen (6) demonstrated that CFA alone could cause the development of circulating brush border antibody. He also demonstrated that the antibody passively administered to the rat would lead to diffuse granular deposition of $\mathrm{IgG}$ along glomerular capillary walls (6). We have furthered the observations of Miettinen.

We wished to study tubular effects of autoimmune-induced interstitial disease before severe glomerular changes developed. We studied animals 12-14 days after initial injection which is prior to the development of proteinuria or glycosuria (16). We have shown that animals injected only once with a TBM preparation plus CFA will develop anti-TBM antibody. These animals will have diminished BBMV uptake of glucose measured over time, and the kinetics of uptake (both Vmax and apparent $\mathrm{Km}$ ) is also diminished. However, when the adjuvant alone is injected, a similar diminution in glucose uptake by BBMV is seen. The effect of in vivo adjuvant is demonstrable after a single injection.

Histologic evaluation shed no light on the mechanism by which adjuvant might influence BBMV uptake of glucose. No deposition of IgG was seen in control or CFA-injected animals (groups 1,2, and 3), whereas injection with TBM plus adjuvant routinely led to $\operatorname{IgG}$ deposition along renal TBM. Light and electron microscopy revealed no cellular infiltration by neutrophils or lymphocytes. Thus, a clear immune-mediated mechanism for diminished BBMV glucose uptake could not be found. We did not assay for circulating brush border antibody or immune complexes. However, no histologic evidence was present for immune-mediated brush border disease.

Miettinen (6) discussed possible mechanisms for adjuvantinduced brush border antibody. These included: 1) cross-reactivity between brush border and proteins in CFA; 2) skin antigen due to injection which cross-reacts with brush border; and 3) polyclonal activation of B-cells. Given the lack of histologic or immune fluorescent changes in our animals, the above mechanisms do not seem likely. CFA may be serving as a tubular toxin. We were unable to show a change in the urinary excretion of glucose in animals receiving adjuvant. However, glucose is avidly reabsorbed by the proximal tubule (19), and the changes induced by adjuvant, although statistically significant at the brush border level, may not lead to significant glycosuria after just one injection.

Two more points deserve comment. First, it is conceivable that CFA may affect glucose transport and no other transport system. Although we did not test this hypothesis directly, it seems quite unlikely that CFA could specifically change the brush border membrane glucose transporter. Second, it is also possible that our findings are peculiar to the L-BN rat. L-BN or Brown Norway rats were employed frequently in the study of immunemediated disease, particularly the study of immune-mediated tubulointerstitial disease. The induction of anti-TBM disease in rats has succeeded in L-BN and Brown Norway rats, but not in Lewis, Wistar-Furth, and others (18). Therefore, the L-BN or Brown Norway rat is likely to continue to be used in studies employing active immunization to produce immune-mediated renal disease. If so, adjuvant injection alone must be used as a control in any experiments studying functional effects in immune-mediated renal disease.

In conclusion, we found that the in vivo administration of CFA led to in vitro (BBMV) changes in glucose transport. These changes occurred after a single administration of CFA. The exact mechanism by which CFA alters glucose transport is unclear. Studies of the functional changes caused by immune-mediated disease induced by CFA must take into account changes created by adjuvant alone.

Acknowledgments. J. Freimuth and W. Humboldt provided editorial assistance.

\section{REFERENCES}

1. Freund $J 1951$ The effect of paraffin oil and mycobacterium on antibody formation and resensitization. Am J Clin Pathol 21:645-656

2. McCluskey RT, Klassen J 1973 Immunologically mediated glomerular tubular and interstitial renal disease. N Engl J Med 288:564-570

3. McCluskey RT, Colum RB 1978 Immunologic aspects of renal tubule and interstitial diseases. Ann Rev Med 29:191-203

4. Steblay RW 1962 Glomerulonephritis induced in sheep by injection of heterologous glomerular basement membrane and Freund's adjuvant. J Exp Med 116:253-271

5. Steblay RW, Rudofsky U 1968 Autoimmune glomerular nephritis induced in sheep by injection of human lung and Freund's adjuvant. Science 160:204 206

6. Miettinen A 1982 Nephritogenic antibodies against kidney brush border glycoproteins in rabbits injected with Freund's adjuvant. Lab Invest 47:67-75

7. Booth AG, Kenny AJ 1974 A rapid method for the preparation of microvilli from rabbit kidney. Biochem $\mathrm{J}$ 142:575-581

8. Chesney RW, Gusowski N, Friedman Al 1983 Renal adaptation to altered dietary sulfur amino acid intake occurs at luminal brush border membrane. Kidney Int 24:588-594

9. Lowry DH, Rosebrough NJ, Farr L, Randall RJ 1951 Protein measurement with Folin phenol reagent. J Biol Chem 193:265-275

10. Hartree A 1972 A modification of the Lowry method that gives a linear photometric response. Anal Biochem 48:422-429

11. Post RL, Sen AK 1981 Sodium- and potassium-stimulated ATPase, Vol 20. In: Estabrook RW, Pullman ME (eds) Methods in Enzymology. Academic Press, New York, pp 762-768

12. Leuer JE 1980 The use of membrane vesicles in transport studies. CRC Crit Rev Biochem XX: 187-246

13. Ryan TA, Joiner BL, Ryan BF 1982 Minitab Reference Manual. Minitab Project Penn State University

14. Glassock RJ, Edgington TS, Watson JI, Dixon FJ 1968 Autologous immune complex nephritis induced with renal tubular antigen. II. The pathogenic mechanism. J Exp Med 127:573-582

15. Lehman DH, Wilson CB, Dixon FJ 1974 Interstitial nephritis in rats immunized with heterologous tubular basement membrane. Kidney Int 5:187nized

16. Sugisaki T, Klassen J, Milgrom F, Andres GA, McCluskey RT 1973 Immunopathologic study of an autoimmune tubular and interstitial disease in Brown Norway rats. Lab Invest 28:658-671

17. Park EK, Noble B, Andres G, Hong SK 1984 Impaired proximal tubule function in Heymann nephritis. Fed Proc 43:543

18. Park EK, Noble B, Andres G, Hong SK 1983 Proximal tubule function in chronic serum sickness glomerulonephritis in rats. Fed Proc 42:523

19. Silverman M 1976 Glucose transport in the kidney. Biochim Biophys Acta 457:303-321

20. McCluskey RT, Andres GA, Klassen J 1976 Immunologically mediated tubular and interstitial renal disease. Proceedings of the 6th International Congress of Nephrology, Florence, Italy, 1975. Karger, Basel, pp 530-541 